\title{
Using Parallelism in Experimenting and Fine Tuning of Parameters for Metaheuristics ${ }^{\star}$
}

\author{
Maria Blesa and Fatos Xhafa \\ Universitat Politècnica de Catalunya \\ C6 Campus Nord, E-08034 Barcelona, Spain \\ $\{$ mjblesa,fatos\}@lsi.upc.es
}

\begin{abstract}
We show that parallel implementations of metaheuristics are efficient tools for both experimenting and fine tuning of parameters.
\end{abstract}

\section{Introduction}

Metaheuristics were introduced in the last two decades as a new kind of approximate algorithms for solving combinatorial optimization problems which combine heuristic methods with higher level frameworks [5]. Their implementation is usually a complex task, since they involve three main concepts: (1) Main method, (2) internal/external heuristics, and (3) setting of search parameters.

Measuring the performance of a metaheuristic implementation requires testing on a large set of instances and on real world instances usually of big and very big size. Moreover, finding of right values for the search parameters of the metaheuristic is almost indispensable for the success of the metaheuristic implementation. Considerable efforts have been done by researchers and practitioners to provide, on the one hand, a methodology and rigorous basis for experimental evaluation of heuristics [112] and, on the other, to find efficient approaches for fine tuning of parameters such as developing specific software [1, use of experimental design [7] and self-adaptive procedures [10].

We address the issue of using parallel implementations as a mean for efficient experimenting and fine tuning of parameters for metaheuristics. Our proposal is based on two parallel models and, to illustrate our proposal, we have applied it in experimenting and fine tuning of parameters for the Tabu Search method applied to the 0-1 Multidimensional Knapsack problem. High quality solutions as compared with best known up-to-date results for the problem are obtained.

\section{Parallel Models for Experimenting and Fine Tuning}

Parallelism has been usually used to reduce computation times. For our purpose we describe here two simple parallel models: the Independent Runs (IR) and

\footnotetext{
* Partially supported by the CICYT Project TIC2002-04498-C05-03 (TRACER) and by the Catalan Research Council of the Generalitat de Catalunya (grant no. 2001FI-00659). For a longer version of this work, see [4].
} 
the Independent Runs with Autonomous Strategies (IRAS). Although closely related, they are used here with different objectives: the IR model is intended for experimenting while IRAS model for the fine tuning of parameters.

In the Independent Runs model (IR) there is a coordinator processor sending the problem instance and parameters' setup and receiving the results, and each processor runs the same instance of the program. Observe that this model make sense as far as the program is non-deterministic. This is precisely the case of metaheuristic implementations which take randomized or probabilistic decisions. Running the same implementation in $p$ different processors leads to exploring different areas of the search space and it is equivalent to performing $p$ sequential executions, thus scaling down the experimentation time with a factor of up to $p$. For this reason, this model is then very suitable for experimental evaluation of metaheuristic implementations. The Independent Runs with Autonomous Strategies (IRAS) can be seen as a special case of the IR model in which the processors are given, additionally, a strategy to be used for its own search. A strategy is defined as an $m$-tuple $\left\langle\right.$ parameter $_{1}, \ldots$, parameter $\left._{m}\right\rangle$, where each parameter $_{i}$ is a different parameter of the metaheuristic. For each processor proc $_{i}$, the coordinator processor proc 0 computes a strategy $S_{i}$, and then sends it together with the problem instance to the processor proc $_{i}$. Clearly, using this parallel model we can efficiently make the fine tuning of parameters.

Our implementation of both parallel models is fully generic and independent of the (sequential) metaheuristic implementation at hand. This is achieved through a careful class design and implementation in $\mathrm{C}++$ using the MPI as a communication library. The class Solver_LAN will be be in charge of running the parallel program while the sub-classes Solver_IR and Solver_IRAS will implement specifically the task of coordinator and slave processors. Solver_Seq denotes the sequential implementation of the metaheuristic, through which we can declare an instance of such implementation and run the main method. There is a one-to-one relationship between Solver_LAN and Solver_Seq since the former will use instances of the latter as a black-box. The classes Instance, Setup, Strategy and Solution represent the problem instance data, parameters, strategy and a feasible solution to the problem, respectively. Those entities are problem-dependent and will be implemented according to the problem at hand. The parallel program provides their interfaces and hence can use them as black-boxes. Any of the models is run via the method run () of the corresponding class Solver_IR or Solver_IRAS. This generic way of designing and implementing the framework has important benefits, like genericity and reusability. Once the execution is finished, different information of the search process can be accessed, e.g., the best solution found or the time required to find it.

\section{Tabu Search for the 0-1 Multidimensional Knapsack}

To illustrate our proposal, we implement the Tabu Search (TS) for the 0-1 Multidimensional Knapsack problem both in the IR model (which is intended for experimenting) and the IRAS model (which is intended for the fine tuning.) 
Table 1. Numerical values for the parameters.

\begin{tabular}{cccc}
\hline nb_iterations & independent_runs & tabu_list_size & max_neighbors \\
$\begin{array}{c}100 n \text { (small and middle) } \\
1000 n \text { (big size) }\end{array}$ & 2 & {$[3 \ldots 15]$} & full exploration \\
\hline nb_best_sols & nb_intensifications & history_rep & nb_diversifications \\
{$[10 \ldots 15]$} & $\simeq 10$ & $80-95 \%$ & $\simeq 10$ \\
\hline
\end{tabular}

Table 2. Results for the 0-1MKNP. Best and average costs obtained over 20 executions, respectively. The 7 th column is the deviation of the sample wrt. the average. The last two columns indicate the number of iterations performed and time spent on it.

\begin{tabular}{l|rrc|rrrrr}
\hline \multicolumn{1}{c|}{ Instance } & $n$ & $m$ & Optimum & Best cost & Avg. cost & deviation & \multicolumn{2}{r}{ Iters. time (s) } \\
\hline KNAP15 & 15 & 10 & 4015 & 4015 & 4014.1 & 0 & 3000 & 3.6 \\
KNAP20 & 20 & 10 & 6120 & 6120 & 6120.0 & 0 & 1600 & 2.5 \\
KNAP50 & 50 & 5 & 16537 & 16520 & 16441.0 & 0.001 & 10000 & 21.3 \\
\hline SENTO1 & 60 & 30 & 7772 & 7772 & 7772.0 & 0 & 5000 & 53.5 \\
SENTO2 & 60 & 30 & 8722 & 8722 & 8720.6 & 0 & 5000 & 55.5 \\
\hline OR10x100-00 & 100 & 10 & 23064 & 22478 & 22360.4 & 0.025 & 85629 & 600 \\
OR10x250-00 & 250 & 10 & 59187 & 56213 & 55945.6 & 0.050 & 55132 & 900 \\
OR10x500-00 & 500 & 10 & 117726 & 111773 & 111486.7 & 0.051 & 35487 & 1200 \\
OR30x100-00 & 100 & 30 & 21946 & 21614 & 21520.7 & 0.015 & 31615 & 600 \\
OR30x250-00 & 250 & 30 & 56693 & 54711 & 54534.6 & 0.035 & 15215 & 900 \\
OR30x500-00 & 500 & 30 & 115868 & 111272 & 110942.4 & 0.040 & 10459 & 1200 \\
\hline
\end{tabular}

Tabu Search 9] belongs to the family of local search algorithms but here the search is done in a guided way in order to overcome the local optima. The search process tries to avoid cycling by forbidding or penalizing moves which take the solution, in the next iteration, to solutions previously visited (called tabu). To this aim, TS keeps a tabu list which constitutes the tabu search memory. The role of the memory can change as the algorithm proceeds. At initialization the goal is to make a coarse examination of the solution space and further on the search is focused to produce local optima solutions in a process of intensification or make a diversification in order to explore new regions of the solution space.

The NP-hard 0-1 Multidimensional Knapsack problem (0-1MKNP) consists in selecting a subset of $n$ given objects in such a way that the total profit of the selected objects is maximized while a set of knapsack constraints are satisfied. The $0-1 \mathrm{MKNP}$ problem can be stated as: maximize $c \cdot x$, subject to: $A x \leq b$, $x \in\{0,1\}^{n}$, where $c \in \mathbb{N}^{n}, A \in \mathbb{N}^{m \times n}$, and $b \in \mathbb{N}^{m}$. The binary components $x_{j}$ of $x$ are decision variables: $x_{j}=1$ if the object $j$ is selected, and $x_{j}=0$ otherwise. The profit associated to $j$ is denoted by $c_{j}$. Each $A_{i} x \leq b_{i}$ is a capacity constraint.

Parameters involved, fine tuning and computational results. Five parameters define the $0-1 \mathrm{MKNP}$ : the number of objects $n$, the number of constraints $m$, the profits of the objects $c \in \mathbb{N}^{n}$, the matrix of constraints $A \in \mathbb{N}^{m \times n}$, and the capacities $b \in \mathbb{N}^{m}$. Every fixed set of values for these parameters defines an instance of the problem and, according to them, instances can be easier or harder to solve. This is an important feature to consider when studying the robustness and the performance of an algorithm. The basic parameters controlling TS are 
concerned with stopping conditions (nb_iterations, and independent_runs) and the influence of the historical search memory (tabu_list_size). Other parameters control the search process, specially the neighborhood exploration (max_neighbors), the diversification (history_rep and nb_diversifications), and the intensification (nb_best_sols and nb_intensifications). All those parameters are mutually and strongly dependent. For the success of the method, appropriate values for those parameters have to be find. We have tuned the Tabu Search parameters by using the IRAS model introduced above.

After tuning these parameters (see Table 1), we have run the 0-1MKNP implementation in a cluster of computers AMD K6-11 with $450 \mathrm{MHz}$ processors and $256 \mathrm{Mb}$ of memory (see 4]). To obtain some statistical significance about the robustness of the algorithm, the same instance should be run several times with the same parameters setting and average results should be provided. We test small $(n \leq 50)$, middle-sized $(50<n \leq 100)$ and big instances $(100<n \leq 500)$ taken from the literature 863 . Since our aim is to test how does our generic implementation and parallel fine tuning of parameters behave, we have chosen instances for which the optimum value (obtained through computationally expensive exact methods) is known (see Table 21). The low values on the deviation of the cost of our solutions from the optimum shows both that the values of the parameters that we found through our approach are appropriate, and also the robustness of our approach in the sense that the values we found for the parameters perform very well for a large set of different instances.

\section{References}

1. B. Adenso-Diaz and M. Laguna. (2002). Fine tuning of Algorithms Using Fractional Experimental Designs and Local Search. Submitted.

2. R.S. Barr, B.L. Golden, J. Kelly, W.R. Stewart, M.G.C. Resende. (2001) Designing and Reporting Computational Experiments with Heuristic Methods. Journal of Heuristics, 1(1):9-32.

3. J.E. Beasley. (1990). OR-Library: Distributing Test Problems by Electronic Mail. Journal of the Operational Research Society, 41:1069-1072.

4. M. Blesa and F. Xhafa. (2003). Using Parallelism in Experimenting and Fine Tuning of Parameters for Metaheuristics. Technical Report no. LSI-03-56-R, UPC.

5. C. Blum and A. Roli. (2003). Metaheuristics in combinatorial optimization: Overview and conceptual comparison. ACM Computing Surveys, 35(3):268-308.

6. C. Cotta and J.M. Troya. (1998). A Hybrid Genetic Algorithm for the 0-1 Multiple Knapsack Problem. In Artificial Neural Nets and Genetic Algorithms, chapter 3, pp. 251-255. Springer-Verlag.

7. S.P. Coy, B.L. Golden, G.C. Runer, E.A. Wasil. (2000). Using Experimental Design to Find Effective Parameter Settings for Heuristics Journal of Heuristics, 7:77-97.

8. A. Freville and G. Plateau. (1990). Hard 0-1 multiknapsack test problems for size reduction methods. Investigation Operativa, 1:251-270.

9. F. Glover and M. Laguna. (1997). Tabu Search. Kluwer Academic Publishers.

10. J. Kivijärvi, P. Fränti and O. Nevalainen. (2003). Self-Adaptive Genetic algorithm for Clustering. Journal of Heuristics, 9:113-129.

11. R.L. Rardin and R. Uzsoy. (2001). Experimental Evaluation of Heuristic Optimization Algorithms: A Tutorial. Journal of Heuristics, 7:261-304. 